
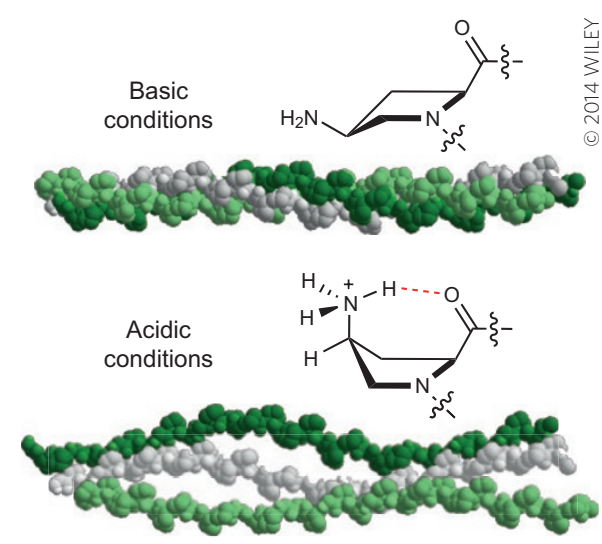

structure that underpins its function in ligaments, tendons and skin. This fibrous superstructure is built on triple helices and these helices are stabilized, in part, by the hydrogen bonding enabled by proline and hydroxyproline residues in the polypeptide chain. Unnatural proline-derived residues - which alter the patterns of hydrogen bonding within, and steric demands on, the helix - can be used to probe the stability of these helices and investigate collagen's conformational properties.

Now, Helma Wennemers and co-workers at ETH Zürich have used a proline derivative in collagen model peptides to reversibly induce and disrupt the folding of a triple helix. Upon protonation, the ring conformation of ( $4 S)$-aminoproline flips and a new transannular hydrogen bond is formed. This change in conformation and hydrogen bonding can destabilize the helix, but the extent to which it does so depends on the location of the proline derivative in the peptide chain. Wennemers and colleagues found that in one position, the additional steric bulk of (4S)-aminoproline -

compared with the proline residue it replaced - destabilized the helix under both basic and acidic conditions.

By instead replacing an adjacent

hydroxyproline residue with

(4S)-aminoproline, they found that, under basic conditions, the helix remained as stable as the natural form. However, on protonation of the amine group, the repulsion between adjacent ammonium cations in the helix, and the rearrangement of hydrogen bonds, weakens the self-assembly. The team took advantage of this to reversibly fold and unfold the triple helix by changing $\mathrm{pH}$. Although adding acid destabilizes the helix sufficiently to 'unzip' it in only six minutes, refolding induced by addition of base - takes much longer. This mismatch is similar to thermally induced denaturing and refolding, which suggests that the mechanism of folding is similar to that already known.

\section{ENZYME CATALYSIS}

\section{Colourful conversion}

Angew. Chem. Int. Ed. http://doi.org/ f2tmj7 (2014)

Chiral amines are found in numerous pharmaceuticals and bioactive natural products. As a result, methods for their asymmetric synthesis are in high demand. Reductive aminations to produce chiral primary amines - in which a prochiral ketone is combined with an amine donor - were identified by a recent round-table meeting of pharmaceutical producers as an aspirational target for green chemistry research. Several classes of enzyme have been shown capable of mediating such reactions and have been applied at both the discovery and manufacturing scale.

Now, Anthony Green and Nicholas Turner from the University of Manchester in collaboration with Elaine O'Reilly from Manchester Metropolitan University have reported the use of an amine donor that could dramatically enhance the efficiency of reductive aminations catalysed by $\omega$-transaminases as well as facilitate the screening process used to identify active enzymes. The key to achieving useful conversions in these reactions is the displacement of an equilibrium that usually favours the starting materials, and several approaches to this have been developed. At the discovery laboratory level, the use of excess alanine as the amine donor, combined with a second enzyme to remove the pyruvate by-product, has been successful. On larger scales, the expense makes such an approach unfavourable and excess isopropylamine has been used as donor, with the by-product - in this case acetone - removed by evaporation. The researchers identified ortho-xylylenediamine as a particularly effective amine donor. Just one equivalent of it is required to obtain high conversions with a variety of ketone starting materials, and use of only 1.5 equivalents gave $73 \%$ conversion of the challenging substrate 1-indanone.

The by-product from this donor is removed from the equilibrium by cyclization followed by tautomerization to form isoindole. This then undergoes a spontaneous polymerization to produce a highly coloured product, which acts as a built-in detection system for conversion. Green, Turner and O'Reilly went on to show that this method of detection could facilitate the development of high-throughput screens for the identification of new enzymes.

$S D$

Written by Stephen Davey, Claire Hansell and $\mathrm{CH}$ Russell Johnson.

\title{
blogroll
}

\section{Getting started}

Perseverance, soft skills and selfreflection are needed for a career in science.

Between 1996 and 2011, fewer than 1\% of scientists published a paper each year, but their names appeared on just over $40 \%$ of all papers (http://go.nature.com/ JzYHyw). It's a long and winding road to join that $1 \%$ and writing at Aidan's Aviary, Aidan Horner lists (http://go.nature. com/5dWjfK) all his rejections, from publications to positions, in his negative $C V$. This is a useful reminder that one can go through many failures before success. Never give up, never surrender.

Odyssey, at Pondering Blather, realizes (http://go.nature.com/AvlTkv) that a good scientific training alone is not enough and that a lot more is needed to set up and run a successful lab, including managing (lab budgets and people), teaching and grant writing. How and when to teach such soft skills is a critical issue and is largely underestimated and poorly recognized. Among these skills, peer-review is still essential to the advancement of science, and Alexis Verger at An Infinity of Hypotheses, offers his reviewer oath (http://go.nature.com/lg4pNu). Whether peer-review should be anonymous or not prompts much debate, but the rest of Verger's coda, including 'Review unto others as you would have them review unto you', should be engraved on the floor of every lab.

Finally, Acclimatrix, writing at Tenure, She Wrote, ponders which kind of mentor she wants to be (http://go.nature.com/ PhTfDn). Funny? Hard? Motherly? Badass? "I want to create a strong lab culture [...] that results not only in strong bonds, but strong science," she writes. Don't we all? The PI plays a critical role in driving the group and mentoring the students, and reflecting on what you want to achieve surely pays off in the long term. "I'll let you know how that goes," Acclimatrix wrote last year. Time for an update?

Written by Sylvain Deville, who blogs at http://sylvaindeville.net 